\title{
Outcome of Cardiac ECMO in Children Less than 1 Year Age Undergoing Corrective Cardiac Surgery
}

\author{
Sarvesh Pal Singh ${ }^{1}$ Sanjoy Sengupta ${ }^{1}$ Manoj Kumar Sahu ${ }^{1}$ Sachin Talwar ${ }^{1}$ \\ Velayoudham Devagourou' Palleti Rajashekar ${ }^{1}$ Ujjwal Kumar Chowdhury ${ }^{1}$ \\ 1Department of Cardio-Thoracic and Vascular Surgery, \\ Cardio-Thoracic Sciences Center, All India Institute of Medical \\ Sciences, New Delhi, India \\ Address for correspondence Sarvesh Pal Singh, DM, All India

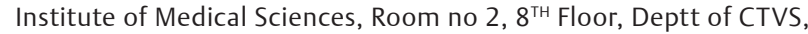 \\ C N Center, New Delhi, 110029, India \\ (e-mail: sarveshpal.singh@gmail.com).
}

J Card Crit Care:2020;4:25-32

\begin{abstract}
Extracorporeal membrane oxygenation (ECMO) has emerged as an effective mechanical support device to support both respiratory and cardiac function following cardiac surgery. The use of ECMO is more common among children who undergo complex surgical procedures. The objective of this study was to observe the outcomes of children subjected to central venoarterial (VA) ECMO after congenital cardiac surgery and identify the factors influencing survival in these children. Twenty children, below the age of 1 year, who underwent corrective cardiac surgery and were placed on ECMO support within first 48 hours of biventricular repair, from July 2018 to June 2019, were included in this study. ECMO was initiated either in the operating room (either preoperative decision or unable to come off cardiopulmonary bypass [CPB]) or in the intensive care unit (low-cardiac output syndrome). The maintenance and weaning from ECMO was done as per institute protocol. At the initiation of ECMO, the flows were maintained between 100 and $150 \mathrm{~mL} / \mathrm{kg} / \mathrm{min}$. The flows were decreased if it was possible to maintain normal perfusion with lesser flows. Out of 20 children, 12 children were less than or equal to 2 months of age. The 30 -day survival was $45 \%$ and all the children were alive at 4 months after discharge from hospital. A negative cumulative fluid balance (CFB) of more than $50 \mathrm{~mL} / \mathrm{kg}$ in the first 48 hours, decreasing lactate and inotropic

Keywords

- cardiac

- children

- ECMO

- outcome

- surgery score, and improving ventricular function in the first 3 days of ECMO were associated with improved survival. The following factors were correlated with decreased survival: aortic cross-clamp time (AOCX T) more than 106 minutes, higher initial ECMO flows (75 vs. 52\%), ECMO duration more than 4 days, increased transfusion of blood and blood products, urine output $<1.06 \pm 0.81 / \mathrm{kg} / \mathrm{h}$ (in the first $48 \mathrm{~h}$ ), and increasing trend of C-reactive protein (CRP) and erythrocyte sedimentation rate (ESR).
\end{abstract}

\section{Introduction}

Extracorporeal membrane oxygenation (ECMO) has emerged as an effective mechanical support device to support both respiratory and cardiac function following cardiac surgery. ${ }^{1}$ Overall, only a small fraction of children are supported with ECMO following cardiac surgery. The use of ECMO is more common among children who undergo complex surgical procedures. ${ }^{2}$ ECMO is now considered a useful therapeutic
DOI https://doi.org/

$10.1055 / \mathrm{s}-0040-1713547$

ISSN 2457-0206. modality in children developing postcardiotomy myocardial dysfunction and low-cardiac output syndrome. Children who develop low-cardiac output syndrome in intensive care unit (ICU) and are unresponsive to conventional management (optimizing preload, increased inotropic support and appropriate afterload manipulation) are suitable candidates for cardiac ECMO. Less commonly, ECMO is used as a support modality when it is difficult to wean children from cardiopulmonary bypass after cardiac surgery. With an overall 
increase in experience, the early institution of ECMO support has been correlated with improved survival, less end organ damage, and less morbidity. In small children, survival rates up to $50 \%$ have been achieved by instituting ECMO prior to the occurrence of multiple organ failure. The overall survival of patients on ECMO as a bridge to recovery and transplantation is $54.5 \%{ }^{3}$ The primary objective of this study was to observe the survival of children subjected to central venoarterial (VA) ECMO after congenital cardiac surgery.

\section{Materials and Methods}

After obtaining ethical clearance from the institute ethics committee and written informed consent from the parents (or guardians), 20 children were enrolled in this study. The reference number for ethical clearance is IECPG186/19.04.2018, RT-11/10.05.2018. This was a prospective observational study conducted over a period of one year (July 2018-2019).

The primary objective of this study was to observe the survival of children subjected to central VA ECMO after congenital cardiac surgery. The secondary objective was to determine whether the following factors affect the survival of children on ECMO: variation in heart rate, variation in blood pressure, cardiac function, serum lactate values, inotropic score, aortic cross-clamp time (AOCX T), ECMO duration, urine output, cumulative fluid balance (CFB) (difference of measured total fluid intake and output), initial ECMO flow as percentage of total flows, sepsis, C-reactive protein (CRP), erythrocyte sedimentation rate (ESR), platelet count, and transfusion of blood and blood products.

Children below the age of 1 year, who underwent corrective cardiac surgery and were placed on ECMO support within first 48 hours of biventricular repair, were included in the study.

Children undergoing emergency surgery, eCPR (children who were not revived after cardiopulmonary resuscitation [CPR] and put on ECMO as extension of CPR) children with single ventricle physiology or diagnosed syndromes, and children in whom ECMO was instituted after 48 hours of corrective cardiac surgery were excluded from this study.

The following subtypes of ECMO were included in this study:

1. ECMO integrated in the operating room-Complex congenital cardiac surgery requiring postoperative support for heart (preoperative decision) or child unable to come off bypass and ECMO continued for hemodynamic support.

2. ECMO instituted in ICU within first 48 hours of surgery presenting with low-cardiac output syndrome (LCOS). LCOS was defined as follows: increased gradient between central and peripheral temperature of more than $10^{\circ} \mathrm{C}$, urine output of less than $0.5 \mathrm{~mL} /$ hour, increased blood lactate levels of more than $10 \mathrm{mmol} / \mathrm{L}, \mathrm{pH}<7.2$ at pCO2 30 to $35 \mathrm{~mm} \mathrm{Hg}$, base deficit $>10 \mathrm{mmol} / \mathrm{L}$, mean arterial pressure $<40 \mathrm{~mm} \mathrm{Hg}$, and inotrope support (four or more of the following): dopamine $10 \mu \mathrm{g} / \mathrm{kg} / \mathrm{min}$; dobutamine
$10 \mu \mathrm{g} / \mathrm{kg} / \mathrm{min}$; adrenaline $0.2 \mu \mathrm{g} / \mathrm{kg} / \mathrm{min}$; noradrenaline $0.2 \mu \mathrm{g} / \mathrm{kg} / \mathrm{min}$; milrinone $0.5 \mu \mathrm{g} / \mathrm{kg} / \mathrm{min}$, ScVO2 less than $60 \%$, and/or $\mathrm{PaO} 2 / \mathrm{FiO} 2<100$ with infiltrates in all quadrants.

\section{Management of Children on ECMO}

\section{Ventilator Management}

After initiation of ECMO, the children were ventilated with pressure regulated volume control (PRVC) mode, and other ventilatory parameters were maintained as follows: fractional inspired oxygen concentration of $40 \%$, tidal volume of 6 to $8 \mathrm{~mL} / \mathrm{kg}$, respiratory rate of 20 per minute, and positive end expiratory pressure (PEEP) of 6 to $8 \mathrm{~cm} \mathrm{H2O}$. The ventilator settings were changed on the basis of compliance of lungs and chest X-ray findings.

\section{Coagulation Management}

The following cutoff values were followed for coagulation parameters in nonbleeding patients: activated coagulation time of 180 to 240 seconds, activated partial thromboplastin time of 50 to 70 seconds, prothrombin time of 18 to 24 seconds, internationalized normal ratio (INR) of $1.5-2.0$, and platelet count $>80,000 / \mathrm{mL}$.

In children with significant bleeding, lower values of activated coagulation time (ACT) 160 to 180 seconds, INR 1.0 to 1.5 , and aPTT 40 to 50 seconds were accepted till the bleeding subsided or ECMO was weaned off, whichever was earlier.

\section{Nutrition}

After 4 hours of institution of ECMO, enteral feeding (expressed breast milk and/or formula feed) through nasogastric tube at $4 \mathrm{~mL} / \mathrm{kg}$ (or less), was administered every 2 hours.

Inotropes-dopamine, dobutamine, milrinone, adrenaline, noradrenaline, and vasopressin were used to maintain heart rate between 100 and $150 \mathrm{bpm}$ (with or without pacing); mean arterial pressure of 30 to $50 \mathrm{~mm} \mathrm{Hg}$, right atrial pressure of 8 to $10 \mathrm{~mm} \mathrm{Hg}$ and urine output of $1 \mathrm{~mL} / \mathrm{kg} / \mathrm{min}$ (with/ without diuretics).

Hematocrit-Ultrafiltration was done with a hemofilter to maintain a hematocrit of 30 to $36 \%$.

ECMO Flows-At the initiation of ECMO, the flows were maintained between 100 and $150 \mathrm{~mL} / \mathrm{kg} / \mathrm{min}$. The flows were decreased if it was possible to establish normal perfusion with lesser flows. The arterial (aortic) line pressure of less than $200 \mathrm{~mm} \mathrm{Hg}$ was accepted.

Sedation-An IV infusion of fentanyl at the rate of 2 to $5 \mu \mathrm{g} / \mathrm{kg} / \mathrm{h}$ was used to provide analgesia and sedation during ECMO. Children were paralyzed intermittently with intravenous rocuronium at a dose of $0.5 \mathrm{mg} / \mathrm{kg}$. Fentanyl dosing was increased with the duration of ECMO.

The rest of the clinical management was done as per ICU protocol. In case of oliguria, IV furosemide was given as bolus, followed by infusion up to a total dose of 3 to $4 \mathrm{mg} / \mathrm{kg} / \mathrm{day}$. Peritoneal dialysis was used in cases of anuria.

The different monitoring modalities used during ECMO are shown in - Table 1 . 
Table 1 Monitoring used during ECMO

\begin{tabular}{|c|c|}
\hline S. No & Continuous monitoring \\
\hline 1 & $\begin{array}{l}\text { Electrocardiography-at least two leads for heart rate, } \\
\text { rhythm, and abnormal changes }\end{array}$ \\
\hline 2 & $\begin{array}{l}\text { Invasive blood pressure and pulsatility of arterial trace } \\
\text { (ejections) }\end{array}$ \\
\hline 3 & Central venous pressure and/or left atrial pressure \\
\hline 4 & Peripheral oxygen saturation \\
\hline 5 & Core and peripheral temperatures \\
\hline 6 & ECMO flows \\
\hline 7 & Pre and postmembrane pressure of oxygenator \\
\hline 8 & Line pressure of arterial (return)cannula \\
\hline 9 & $\begin{array}{l}\text { Ventilator-airway pressure, FiO2, tidal volume, } \\
\text { auto-PEEP }\end{array}$ \\
\hline \multicolumn{2}{|r|}{ Intermittent monitoring 3-4 hourly } \\
\hline 10 & $\begin{array}{l}\text { Arterial blood gas monitoring-pH, } \mathrm{PCO} 2, \mathrm{PaO} 2, \mathrm{BE}_{\text {ect, }} \\
\mathrm{HCO} 3 \text {, lactate, } \mathrm{Hb}, \mathrm{Hct}, \mathrm{O} 2 \mathrm{Hb}, \mathrm{HHb}, \mathrm{Met} \mathrm{Hb}, \mathrm{COHb} \\
\mathrm{Na}^{+}, \mathrm{K}^{+}, \mathrm{Ca}^{2+}, \mathrm{Mg}^{++}, \mathrm{Cl}^{-},{ }^{\mathrm{Ca+}} \text {, blood sugar }\end{array}$ \\
\hline 11 & Abdominal girth \\
\hline 12 & Soakage of surgical dressing \\
\hline \multicolumn{2}{|r|}{ Intermittent monitoring 12 hourly } \\
\hline 13 & $\begin{array}{l}\text { Echocardiography-left and right ventricular function, } \\
\text { valvular regurgitation, gradient across pulmonary and } \\
\text { aortic valves, pulmonary artery systolic pressure and } \\
\text { pericardial effusion }\end{array}$ \\
\hline 14 & Chest X-ray \\
\hline \multicolumn{2}{|r|}{ Intermittent monitoring 24 hourly } \\
\hline 15 & Ultrasonography of lungs \\
\hline 16 & Complete blood count with ESR, CRP \\
\hline 17 & Renal function tests; liver function tests \\
\hline 18 & $\begin{array}{l}\text { Prothrombin time, activate partial thromboplastin time, } \\
\text { international normalized ratio }\end{array}$ \\
\hline
\end{tabular}

Abbreviations: BE, base excess; $\mathrm{COHb}$, Carboxyhemoglobin; FiO2, inspiratory fraction of oxygen; $\mathrm{Hb}$, hemoglobin; $\mathrm{HCO}$, bicarbonate ion; $\mathrm{HHB}$, deoxygenated hemoglobin; Met $\mathrm{Hb}$, Methemoglobin; $\mathrm{O} 2 \mathrm{Hb}$, oxygenated hemoglobin; PaCO2 partial pressure of carbon dioxide in arterial blood; $\mathrm{PaO2}$, partial pressure of oxygen in arterial blood; $\mathrm{pH}$, puissance hydrogen.

\section{Weaning of Children from ECMO}

Weaning was considered in children when following parameters were met: stable heart rate and rhythm, hemodynamically stable on optimal inotropes (any four of the following: dopamine $\leq 5 \mu \mathrm{g} / \mathrm{kg} / \mathrm{min}$; dobutamine $\leq 5 \mu \mathrm{g} / \mathrm{kg} / \mathrm{min}$; adrenaline $\leq 0.1 \mu \mathrm{g} / \mathrm{kg} / \mathrm{min}$; noradrenaline $\leq 0.1 \mu \mathrm{g} / \mathrm{kg} / \mathrm{min}$; milrinone $\leq 0.375 \mu \mathrm{g} / \mathrm{kg} / \mathrm{min}$ ), no active bleeding, arterial blood gases within acceptable limits, lactate $<2.0 \mathrm{mmol} / \mathrm{L}$, chest $\mathrm{X}$-ray within normal limits, normal breath sounds with minimal added sounds, no evidence of lower respiratory tract infection, hematocrit between 30 to $35 \%$, and no severe end organ dysfunction.

To begin weaning; ECMOs flows were decreased in $50 \mathrm{~mL}$ quanta every 3 to 4 hours. The ventilatory parameters were gradually increased in proportion to the decrease ECMO flows to maintain a $\mathrm{pH}$ of $>7.30, \mathrm{PaO} 2>100 \mathrm{~mm} \mathrm{Hg}$ and $\mathrm{PaCO} 2<40 \mathrm{~mm} \mathrm{Hg}$. Ultrafiltration was done at a rate of 1 to $2 \mathrm{~mL} / \mathrm{kg}$ every 2 hours to maintain a hematocrit of 30 to $36 \%$ while maintain a MAP of $>35 \mathrm{~mm} \mathrm{Hg}$. Blood transfusion was done if hematocrit was not achieved in the desired range. Anticoagulation was maintained as described.

The ECMO flows were gradually decreased till a minimal of 50 to $100 \mathrm{~mL} / \mathrm{min}$ flow was left, and then ECMO was stopped progressively over 1 to 2 hours. Serial arterial blood gas analyses and echocardiograms were done after weaning to assess the child repetitively.

\section{Statistical Analysis}

Data was analyzed using Statistical Package for Social Sciences (SPSS) version 21.0 (IBM Corporation, New York, USA). Categorical variables are presented in number and percentage (\%) and continuous variables are presented as mean \pm standard deviation (SD) and median. Normality of data was tested by Kolmogorov-Smirnov test. If the studied parameter did not follow normal distribution, then nonparametric test was used. Quantitative variables like survival, heart rate, mean arterial pressure were compared using independent $t$-test/Mann-Whitney Test (when the datasets were not normally distributed) between the two groups. Qualitative variables were correlated using Chi-Square test/Fisher's exact test. A $P$ value of $<0.05$ was considered statistically significant.

Mauchlys test of sphericity was prepared to check for the assumption, and Greenhouse Geisser value was obtained for determining significance in parametric tests like $t$-test for analyzing heart rate, mean arterial pressure, serum lactate, inotrope score, CRP, ESR, platelet count, hematocrit, and initial ECMO flow.

Similarly, for parameters, where normal distribution could not be demonstrated like AOCX T, urine output, CFB, sepsis, postoperative bleeding, transfusion of packed red blood cells (PRBC), transfusion of fresh frozen plasma (FFP), duration of ECMO, and transfusion of platelets, nonparametric MannWhitney U test was applied.

For qualitative analysis, Fischer's exact test was used for qualitative variables like cardiac function (categorized as mild, moderate and severe) and presence of sepsis.

\section{Results}

Out of 20 children, 8 were female. The children in our study had a median age and weight of 60 days and $3.4 \mathrm{~kg}$, respectively. The most common diagnosis (17 children) was transposition of great arteries (TGA). Out of 20 children, 17 underwent arterial switch operation, 1 truncus arteriosus repair, 1 atrioventricular canal defect (AVCD) repair, and 1 anomalous left coronary artery from pulmonary artery (ALCAPA) repair.

Nine children were weaned off ECMO successfully and all of them were discharged from the hospital. The 30-day survival was $45 \%$ (9/20). All the surviving nine children were followed for a minimum duration of 4 months. The follow-up was $100 \%$ complete and all the children were alive at 4 months after discharge from hospital. One child died 
within 24 hours of surgery and could not be included in statistical analysis for certain parameters.

The following factors were evaluated as determinants of survival in these children: variation in heart rate, variation in blood pressure, cardiac function, serum lactate values, inotropic score, AOCX T, ECMO duration, urine output, CFB (difference of measured total fluid intake and output), initial ECMO flow as percentage of total flows, sepsis, CRP, ESR, platelet count, and transfusion of blood and blood products.

The variation in heart rate (HR) and mean arterial pressure (MAP) was analyzed in 19 children for the first 3 days on ECMO. There was a significant variation in HR over the first 3 days in survivors (D1, D2, D3 146 $\pm 8,149 \pm 12,125 \pm 28$ beats per minute [BPM]) compared with nonsurvivors (D1, D2, D3 $147 \pm 12,143 \pm 17,140 \pm 30 \mathrm{BPM}$; $p$-value 0.001). However, this variation was not statistically significant (-Fig. 1). There was no significant variation in MAP in both survivors and nonsurvivors (D1, D2, D3 $46.6 \pm 6.1,44.14 \pm 7.81,45 \pm 5$ versus $42.3 \pm 10,43.54 \pm 10.26,37 \pm 11 \mathrm{~mm} \mathrm{Hg})$. Left and right ventricular function was evaluated by echocardiography and statistically analyzed only for the first 3 days of ECMO. There was a strong correlation between improving cardiac function and survival on ECMO ( $p$-value 0.001).

As 5 children did not survive for more than 3 days on ECMO, lactate values were studied in 15 children for the first 5 days. The decreasing values of serum lactate $(p<0.05)$ and inotropic score $(p$-value 0.003$)$ significantly correlated with improved survival (-Figs. 2, 3). Increased AOCX T (106 \pm 31.49 vs. $88 \pm 21.69 \mathrm{~min} ; p=0.025)$, longer ECMO duration (more than $4 \mathrm{~d} ; p=0.04$ ) and lower urine output ( $1.06 \pm 0.81$ vs. $1.85 \pm 0.91 \mathrm{~mL} / \mathrm{kg} / \mathrm{h} ; p=0.04)$ in the first 2 days on ECMO was significantly associated ( $p=0.025$ ) with decreased survival (-Figs. 4-6).

A negative CFB of $-58 \mathrm{~mL} / \mathrm{kg}$ (median value) in the first 48 hours on VA ECMO was found to be associated with increased survival (nonsurvivors, $+43 \mathrm{~mL} / \mathrm{kg} ; p=0.00$ )

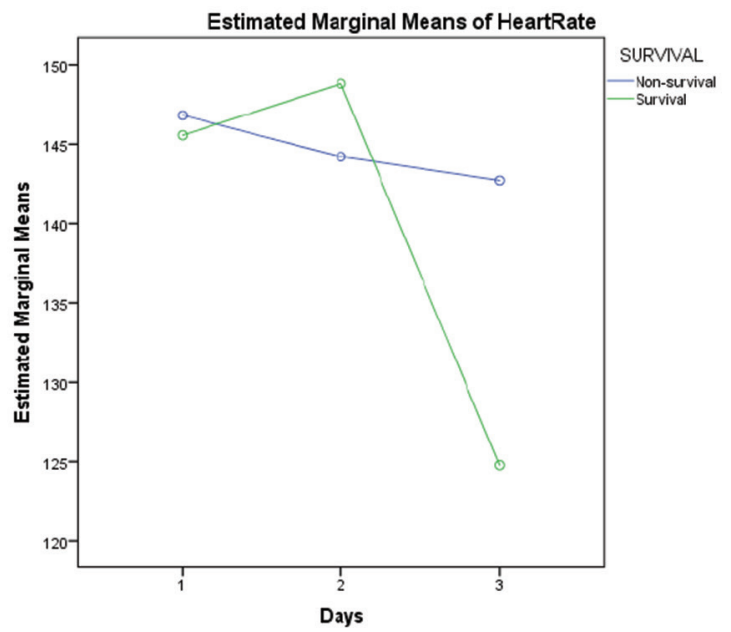

Fig. 1 Graph showing comparison of marginal means of heart rate between survivors and non survivors for first 3 days of ECMO support. Abbreviation: ECMO, extracorporeal membrane oxygenation.
(-Fig. 7). Initial ECMO flow, as percentage of total flows, was also analyzed for all 20 patients. There was a statistically significant correlation between lower initial ECMO flows and survival (52.44 $\pm 8.48 \%$ vs. $75.09 \pm 6.97 \% ; p=0.001$ ) (-Fig. 8).

Platelet counts on day 1, 2 and 3 were analyzed for significance with respect to survival, and it was found that decreasing trend of platelet count was associated with decreased survival ( $p$-value 0.026). Increasing trend of CRP (survival vs. nonsurvival-D1:D2:D3-81.00 vs. 72.23: 64.40 vs. 87.76:75.17 vs. 103.46) and ESR values (survival vs. nonsurvival-D1:D2:D3-18.5 vs. 21:14.55 vs. 25.5:17.55 vs. 27.6) was associated with decreased survival as well (-Figs. 9, 10, 11).

The postoperative bleeding in the first 5 days was significantly higher in non survivors compared with survivors $(137.0 \pm 136.2$ vs. $65.95 \pm 37.97 \mathrm{~mL} / \mathrm{kg} ; p<0.05)$. Similarly, for the first 5 days, there was increased transfusion of PRBC

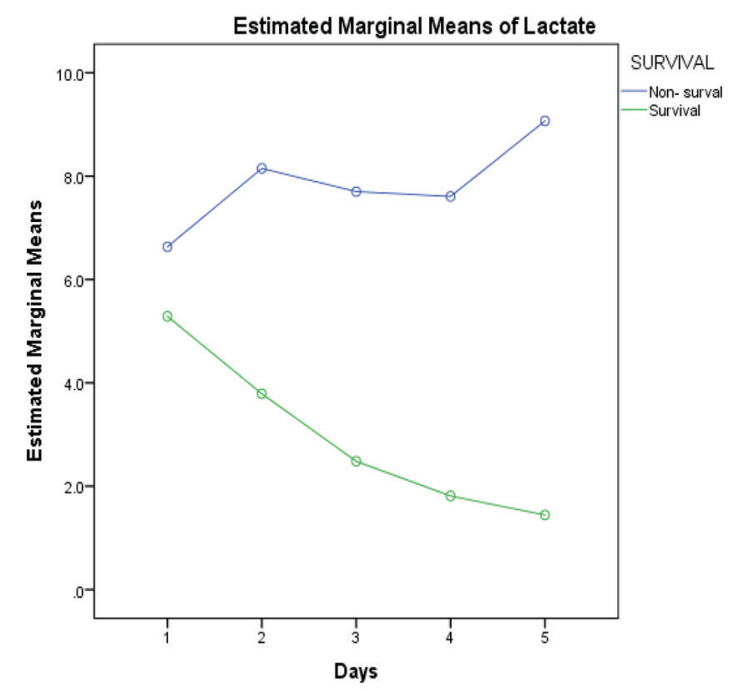

Fig. 2 Showing widely divergent trend of increasing serum lactate in the nonsurvival group versus decreasing serum lactate in the survival group.

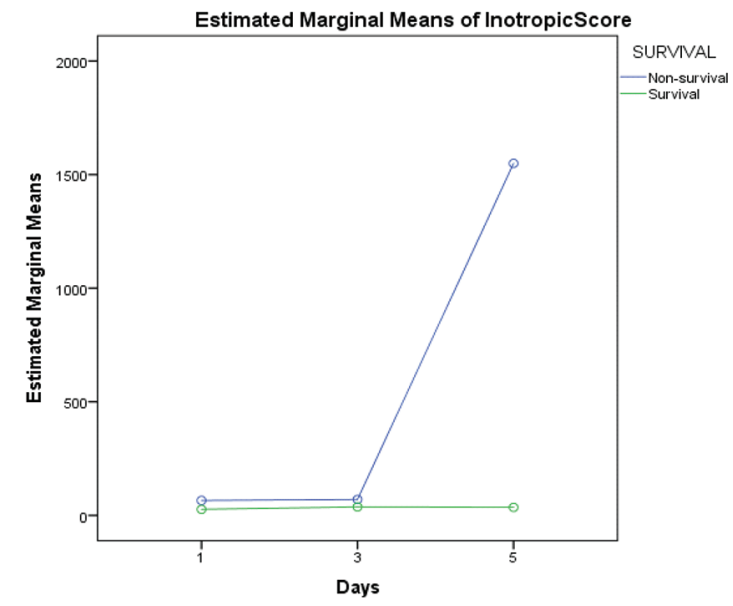

Fig. 3 Showing divergent trend of increasing need of inotrope support in the nonsurvival group from day 3 onward. 


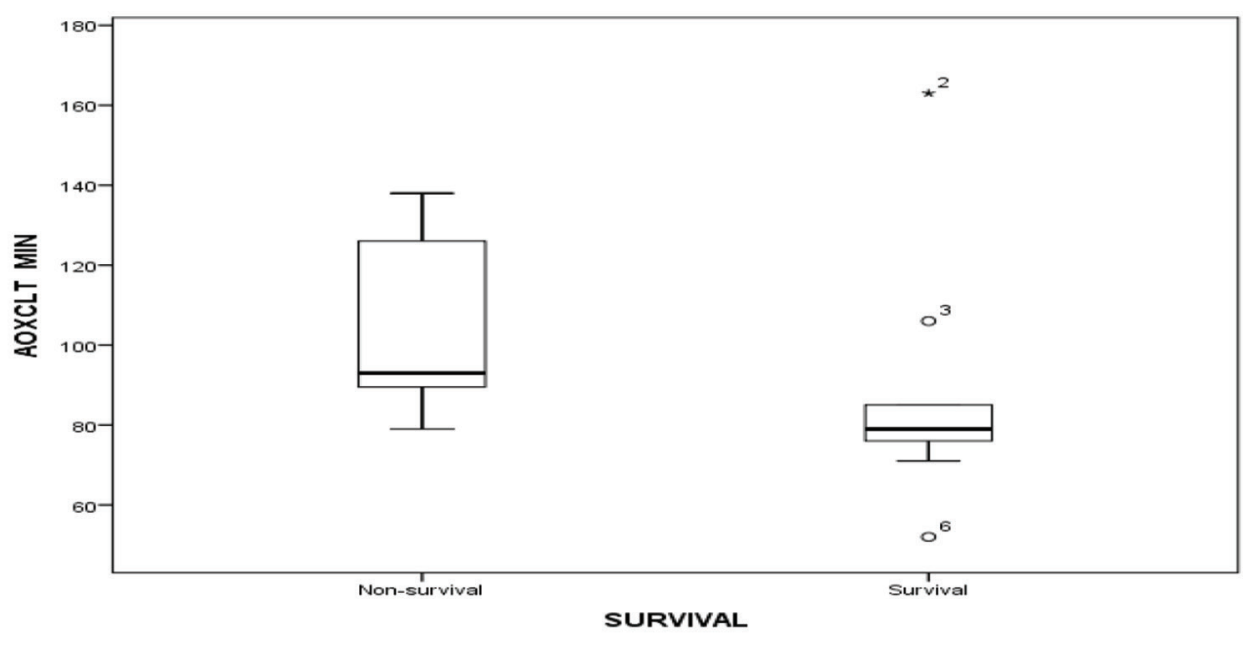

Fig. 4 Showing AOCX T in minutes among survival and nonsurvival group. Abbreviation: AOCX T, aortic cross-clamp time.

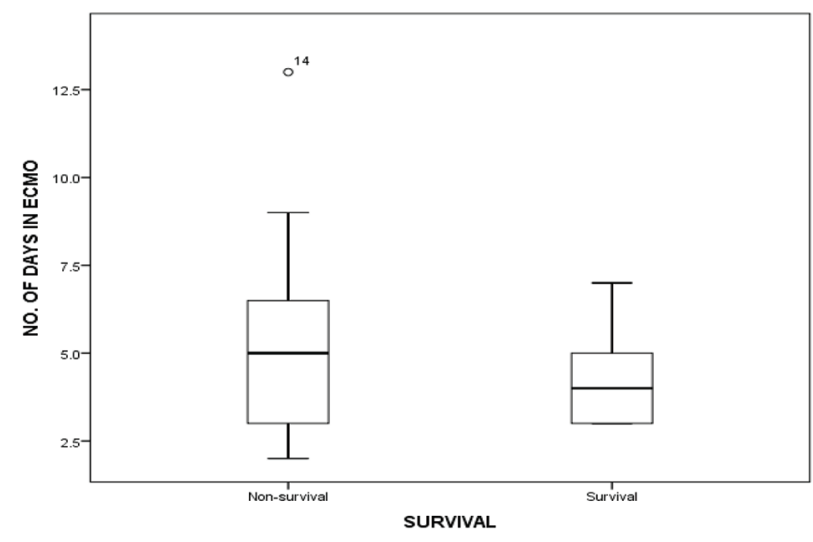

Fig. 5 Showing duration of ECMO run in days amongst survival and nonsurvival group. Abbreviation: ECMO, extracorporeal membrane oxygenation.

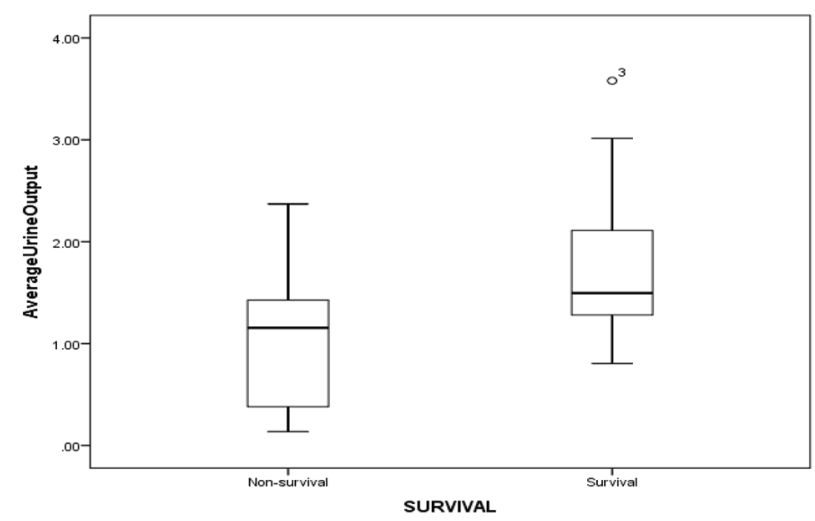

Fig. 6 Showing average urine output in ( $\mathrm{mL} / \mathrm{kg} /$ day) among survival and nonsurvival group.

(135.4 \pm 46.4 vs. $47.85 \pm 60 \mathrm{~mL} / \mathrm{kg} ; p=0.004), \mathrm{FFP}(36.5 \pm 32.8$ vs. $15.16 \pm 14.4 \mathrm{~mL} / \mathrm{kg} ; p=0.03)$, and platelet concentrates $(39.6 \pm 19.31$ vs. $18.56 \pm 10.48 \mathrm{~mL} / \mathrm{kg} ; p=0.016)$ in nonsurvivors compared with survivors.

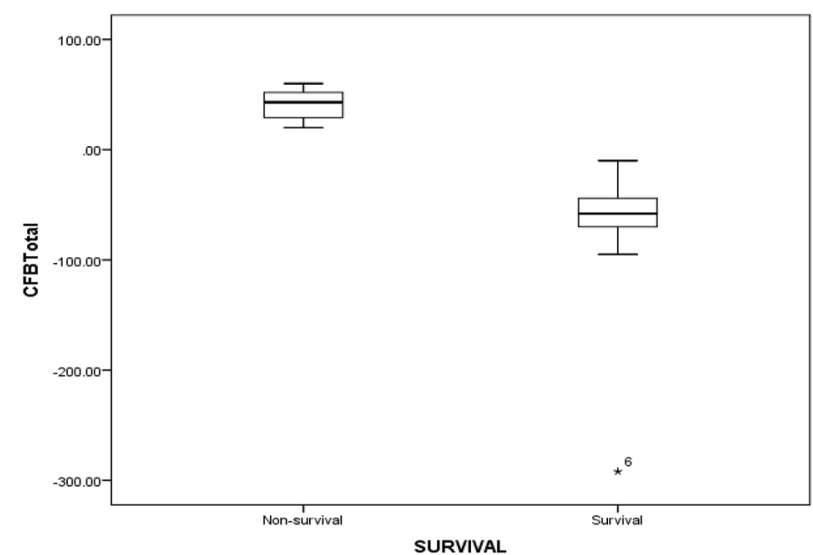

Fig. 7 Showing CFB of first two days on ECMO amongst survival and nonsurvival group. Abbreviations: CFB, cumulative fluid balance; ECMO, extracorporeal membrane oxygenation.

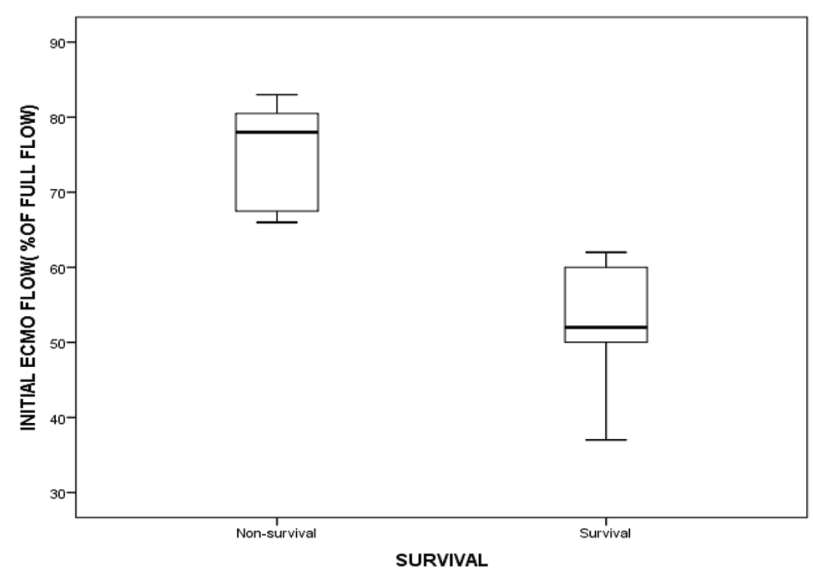

Fig. 8 showing initial ECMO flow (as \% of total full flows) among survival and nonsurvival group. Abbreviation: ECMO, extracorporeal membrane oxygenation.

There was no statistically significant correlation between presence of sepsis and mortality. 


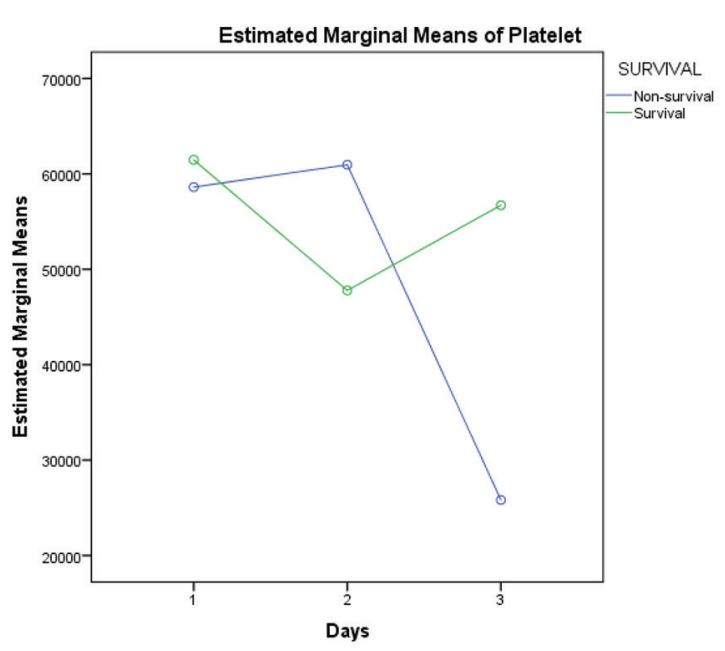

Fig. 9 Graph showing comparison of mean platelet counts between survivors and nonsurvivors for first 3 days of ECMO support. Abbreviation: ECMO, extracorporeal membrane oxygenation.

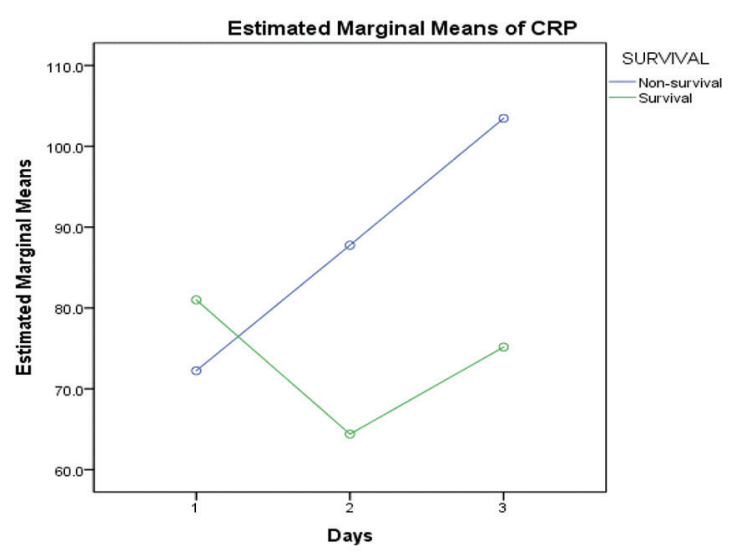

Fig. 10 Showing rising trend of CRP values flow among nonsurvival group. Abbreviation: CRP, C-reactive protein.

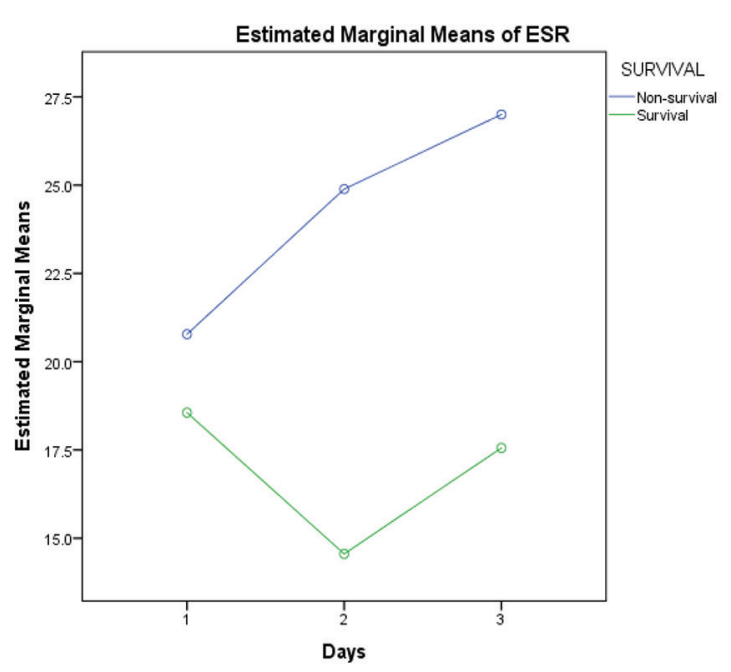

Fig. 11 Showing rising trend of ESR values flow amongst nonsurvival group. Abbreviation: ESR, erythrocyte sedimentation rate.

\section{Discussion}

The use of cardiac ECMO in children has increased over the last few decades. A rise in the number of complex cardiac surgeries in children is the primary cause of increased usage of VA ECMO in children. In the era of personalized medicine, the decision to initiate postcardiac surgery ECMO is individualized on case-to-case basis.

The survival after VA ECMO in children varies between 36 to $48 \%{ }^{4-10}$ The children in our study had a median age and weight of 60 days and $3.4 \mathrm{~kg}$, respectively. Eighty five percent of our study children underwent arterial switch operation (RACHS-4). All the children underwent central (ascending aorta-right atrial) cannulation in the operating room only. The decision to use ECMO was preoperative in 15\% (3/20) children and postoperative in the rest of patients. In our prospective observational study, the 4-month survival was $45 \%(9 / 20)$. After discharge from the hospital, all the children were alive on the first, second and third follow-up of 15 , 30 and 120 days after discharge.

We had prospectively looked into detailed hourly and daily parameters while on ECMO flow which have not been studied previously. There was a significant difference of variation of HR on the days following institution of ECMO to the child, but there was no significant correlation of such variation with respect to survival. However, in survivors, HR significantly decreased over the first 3 days of ECMO. Majority of our children (85\%) underwent arterial switch operation. In TGA, after surgery, the left ventricle is exposed to increased afterload initially. The left ventricle (LV) compensates by mechanism of heterometric regulation. In heterometric regulation, because of LV dysfunction, initial end-diastolic and end-systolic volumes are increased with resultant decrease in stroke volume. Therefore, HR increases to compensate for decreased stroke volume and maintain cardiac output. The purpose of ECMO after arterial switch operation is to train the LV for the increased after load. Hearts which are not able to handle the increased afterloads are supported with ECMO. Slowly, the flows on ECMO are reduced, so that the heart is gradually exposed to the new afterload. Once the LV is accustomed to the new afterload, the LV function increases and end-diastolic and end-systolic volume revert to earlier values. Because the myofibril length is the same as earlier, this compensation is known as homeometric regulation. Due to increase in LV function and stroke volume, the initial increase in heart rate settles to normal values.

Echocardiography is not only a method of need assessment for ECMO, but it also helps in choosing the appropriate mode of support. Echocardiography is a valuable tool for excluding complications like pericardial tamponade, aortic regurgitation, cannula malposition, thrombus, and hypovolemia. ${ }^{11}$ It has a role in everyday assessment of heart to predict the need of further mechanical support or the possibility of successful weaning from ECMO. In our study, we did baseline echocardiography evaluation after initial ECMO setup, followed by serial evaluations of heart every 12 hours during ECMO run. We observed that an improvement in the echocardiographic 
parameters in the first 72 hours, after commencement of ECMO, leads to successful weaning from ECMO and positive outcomes.

With improvement in cardiac function, the lactate levels tend to settle down. The decreasing trend of serum lactate was associated with survival in our study. In a study by Li et al, they observed that early change in lactate values ( 6 and 12 hour) following the initiation of ECMO support can predict in-hospital mortality in postcardiac surgery patients and successful weaning from ECMO as well. ${ }^{2}$ In a case report from our institute, we measured lactate level, calculated lactate clearance at 6 and 18 hour after initiation of ECMO in a child who underwent arterial switch operation for TGA, and concluded that increased lactate clearance predicts successful weaning from ECMO. ${ }^{13}$

A high-peak inotropic score predicted mechanical support in children requiring ECMO support, following fulminant myocarditis. ${ }^{14,15}$ In our study, we found that while on ECMO, decreasing trend of vasoactive inotropic score over the first 5 days was associated with successful weaning and significantly improved survival on VA-ECMO ( $p$ value 0.003 ).

The duration of CPB and AOCX T correlates with the need for VA ECMO and mortality. Cardiopulmonary bypass time of more than 400 minutes and AOCX T of more than 120 minutes were associated with decreased survival. ${ }^{9}$ In our study, even after excluding an exceptionally high-value for AOCX T in nonsurvival group, we found significant correlation between increased AOCX T and decreased survival $(p<0.05)$.

ECMO duration beyond 7 days in children increased the odds of mortality by $12 \%$ for every extra day on VA ECMO, with no survival benefit beyond 10 days. ${ }^{49}$ Hintz et al reported improved survival in neonates who underwent ECMO for less than 3 days. ${ }^{16}$ In our study, four children died within 3 days of ECMO initiation. In the remaining 16 children, we found that increased duration of ECMO (more than 4 days) lead to decreased survival.

Khorsandi et al established that patients on ECMO who develop dialysis-dependent renal failure had higher mortality compared with patients who do not require renal replacement therapy. ${ }^{9}$ Similarly, in another study from United Kingdom, patients requiring renal replacement therapy for renal failure were twice in the nonsurvivors group (20/24) versus the survivors group $(10 / 24){ }^{8}$ In our study, out of 11 children who died, 6 required ultrafiltration after 24 hours of ECMO institution. Whereas in the survival group, there was a significant increase in the urine output over 24 to 72 hours after ECMO initiation. We also found that there was a strong correlation between overall survival and ability to have a negative cumulative fluid balance (NCFB) within first 48 hours of ECMO $(-58 \mathrm{~mL} / \mathrm{kg}$ in the survival group versus $+41 \mathrm{~mL} / \mathrm{kg}$ in the nonsurvival group). The ability to generate a significant negative CFB will depend on an optimal cardiac output, fair renal function, and subsiding systemic inflammatory response to ECMO. Therefore, achievement of a $50 \mathrm{~mL} / \mathrm{kg}$ of negative CFB may indicate general well-being of the child.

In our study, the initial ECMO flow (as a percentage of total flow) correlated with survival. Lesser initial flows were associated with better survival. It is possible that children requiring higher initial flows were sicker and had higher degree of ventricular dysfunction to begin with.

The most recent Extracorporeal Life Support Organization (ELSO) registry report found that 7 to $26 \%$ of neonates placed on ECMO for respiratory and cardiac indications suffered surgical site bleeding. Bleeding complications may occur in up to one-third of children being managed on cardiac ECMO with adverse effect on survival. ${ }^{17}$ The adverse effect of postoperative bleeding on patient survival is evident in our study as well. There are many causes of thrombocytopenia in children supported on cardiac ECMO, for example, mechanical destruction of platelets, dilutional thrombocytopenia, inadequate production (due to extremely sick child), drug-induced thrombocytopenia, and sepsis. In our study, the thrombocytopenia correlated with development of sepsis in the nonsurviving $(5 / 11)$ children. The optimal threshold for transfusion of PRBCs, platelet concentrates (PC), FFP, and cryoprecipitate are not clearly defined to guide clinical decision-making. Also, increased transfusion of blood and blood products is associated with increased mortality. ${ }^{17-20}$ In our study, increased transfusion of PRBCs, FFP, and PC was associated with decreased survival as well.

ECMO induces a sustained systemic inflammatory response syndrome, which is due to exposure to nonphysiological surfaces, surgical trauma, and continuous nonpulsatile flow. ${ }^{21}$ Neonates and young infants are at greater risk because of the disparity between the patient's estimated blood volume (EBV) and the priming volume of the ECMO circuit (2-3 times of EBV of the child). The clinical similarities between systemic inflammatory response syndrome (SIRS) and sepsis make differentiation between them very difficult. Hence, early recognition of sepsis by using biomarkers is difficult, especially in children with ECMO. In our study, increasing trends of both CRP and ESR correlated well with mortality in univariate analysis.

\section{Limitations of the Study}

The sample size of this study is small. The study enrolled both types of ECMO-one instituted in OT and other placed in ICU. Therefore, uniformity of the cohort was not maintained. We did not try to determine exact incidence of neurological deficits during ECMO or the neuropsychological behavior of children after discharge from hospital. We did not correlate the survival with established factors like age and body weight because of small sample size. We did not measure cardiac biomarkers to quantify the amount of myocardial damage.

\section{Conclusion}

A total of $45 \%$ children (9/20) postcorrective cardiac surgery supported with ECMO survived and were discharged from the hospital. All of them were alive at 4 months postdischarge. An increased survival was associated with improved ventricular function during the first 3 days of ECMO, decreased lactate levels and inotropic score, and a negative CFB of more than $50 \mathrm{~mL} / \mathrm{kg}$ in the first 48 hours. The following factors correlated 
with decreased survival: AOCX T more than 106 minutes, initial ECMO flow more than $75 \%$ of total full flow, ECMO duration more than 4 days, urine output $<1.06 \mathrm{~mL} / \mathrm{kg} / \mathrm{h}$ (in the first 48 hours), increased transfusion of blood and blood products, and increasing trend of CRP and ESR.

Conflict of Interest

None declared.

\section{References}

1 Itoh H, Ichiba S, Ujike Y, Kasahara S, Arai S, Sano S. Extracorporeal membrane oxygenation following pediatric cardiac surgery: development and outcomes from a single-center experience. Perfusion 2012;27(3):225-229

2 Bratton SL, Chan T, Barrett CS, Wilkes J, Ibsen LM, Thiagarajan RR. Metrics to assess extracorporeal membrane oxygenation utilization in pediatric cardiac surgery programs. Pediatr Crit Care Med 2017;18(8):779-786

3 Chauhan S, Subin S. Extracorporeal membrane oxygenation, an anesthesiologist's perspective: physiology and principles. Part 1. Ann Card Anaesth 2011;14(3):218-229

4 Gupta P, Robertson MJ, Beam B, et al. Relationship of ECMO duration with outcomes after pediatric cardiac surgery: a multi-institutional analysis. Minerva Anestesiol 2015;81(6): 619-627

5 Agarwal HS, Hardison DC, Saville BR, et al. Residual lesions in postoperative pediatric cardiac surgery patients receiving extracorporeal membrane oxygenation support. J Thorac Cardiovasc Surg 2014;147(1):434-441

6 Alsoufi B, Awan A, Manlhiot C, et al. Does single ventricle physiology affect survival of children requiring extracorporeal membrane oxygenation support following cardiac surgery? World J Pediatr Congenit Heart Surg 2014;5(1):7-15

7 Shah SA, Shankar V, Churchwell KB, et al. Clinical outcomes of 84 children with congenital heart disease managed with extracorporeal membrane oxygenation after cardiac surgery. ASAIO J 2005;51(5):504-507

8 Balasubramanian SK, Tiruvoipati R, Amin M, et al. Factors influencing the outcome of paediatric cardiac surgical patients during extracorporeal circulatory support. J Cardiothorac Surg 2007;2:4

9 Khorsandi M, Davidson M, Bouamra O, et al. Extracorporeal membrane oxygenation in pediatric cardiac surgery: A retrospective review of trends and outcomes in Scotland. Ann Pediatr Cardiol 2018;11(1):3-11
10 Asano M, Matsumae H, Suzuki K, et al. Prognostic risk analyses for postcardiotomy extracorporeal membrane oxygenation in children: a review of early and intermediate outcomes. Pediatr Cardiol 2019;40(1):89-100

11 Bautista-Rodriguez C, Sanchez-de-Toledo J, Da Cruz EM. The role of echocardiography in neonates and pediatric patients on extracorporeal membrane oxygenation. Front Pediatr 2018;6:297

12 Li CL, Wang H, Jia M, Ma N, Meng X, Hou XT. The early dynamic behavior of lactate is linked to mortality in postcardiotomy patients with extracorporeal membrane oxygenation support: A retrospective observational study. J Thorac Cardiovasc Surg 2015; 149(5):1445-1450

13 Singh SP, Chauhan S, Bisoi AK, Sahoo M. Lactate clearance for initiating and weaning off extracorporeal membrane oxygenation in a child with regressed left ventricle after arterial switch operation. Ann Card Anaesth 2016;19(1):188-191

14 Kuraim GA, Garros D, Ryerson L, et al; Western Canadian Complex Pediatric Therapies Follow-up Program. Predictors and outcomes of early post-operative veno-arterial extracorporeal membrane oxygenation following infant cardiac surgery. J Intensive Care 2018;6:56

15 Casadonte JR, Mazwi ML, Gambetta KE, et al. Risk factors for cardiac arrest or mechanical circulatory support in children with fulminant myocarditis. Pediatr Cardiol 2017;38(1):128-134

16 Hintz SR, Benitz WE, Colby CE, Sheehan AM, Rycus P, Van Meurs KP; ELSO Registry. Utilization and outcomes of neonatal cardiac extracorporeal life support: 1996-2000. Pediatr Crit Care Med 2005;6(1):33-38

17 Cashen K, Meert K, Dalton H. Anticoagulation in neonatal ECMO: an enigma despite a lot of effort! Front Pediatr 2019;7:366

18 Muszyinski JA, Reeder RW, Hall MW, et al. Red blood cell transfusion practices in pediatric extracorporeal membrane oxygenation. Crit Care Med 2018;46:e552-e559

19 Tillman B, Gailani D. Inhibition of Factors XI and XII for prevention of thrombosis induced by artificial surfaces. Semin Thromb Hemost 2018;44(1):60-69

20 Saini A, West AN, Harrell C, et al. Platelet transfusions in the PICU: does disease severity matter? Pediatr Crit Care Med 2018;19(9):e472-e478

21 Rungatscher A, Merlini A, De Rita F, et al. Diagnosis of infection in paediatric veno-arterial cardiac extracorporeal membrane oxygenation: role of procalcitonin and C-reactive protein. Eur J Cardiothorac Surg 2013;43(5):1043-1049 